\title{
Evaluation of hypocholesterolemic effect of oleuropein in cholesterol-fed rats
}

\author{
Fatma Hadrich $^{1}$, Asma Mahmoudi ${ }^{1}$, Zouhaier Bouallagui ${ }^{1}$, Ines Feki ${ }^{1}$, Hiroko \\ Isoda $^{2,3}$, Bruno Feve $^{4}$ and Sami Sayadi ${ }^{*}$
}

${ }^{1}$ Environmental Bioprocesses Laboratory, AUF Regional Excellence Pole (AUF-PER-LBP), Sfax Biotechnology Center, P.O. Box 1177, Sfax 3038.

${ }^{2}$ Faculty of Life and Environmental Sciences, University of Tsukuba, Tsukuba, Ibaraki 305-8572, Japan

${ }^{3}$ Alliance for Research on North Africa (ARENA), University of Tsukuba, Tsukuba, Ibaraki 3058572, Japan

${ }^{4}$ INSERM UMR S938, Centre de Recherche Saint-Antoine, F-75012 Paris, France

\section{${ }^{*}$ Corresponding author:}

Environmental Bioprocesses Laboratory, AUF Regional Excellence Pole (AUF-PER-LBP), Sfax Biotechnology Center; P.O. Box 1177, Sfax 3038; Tunisia.

Tel: +216 $74874452 /$ Fax: +216 74874452 .

E-mail address: Sami.sayadi@cbs.rnrt.tn 


\begin{abstract}
Oleuropein, which is the major compound of olive leaves, has been reported to exert several pharmacological properties, including anti-cancer, antidiabetic and antiatherosclerotic activities. The objective of this study was to evaluate the effect of oleuropein on adiponectin level in high cholesterol diet (HCD) induced obesity in rat and the molecular mechanism underlying its activation. Our results showed that orally administered oleuropein $(50 \mathrm{mg} / \mathrm{kg})$ by gavage for 8 weeks decreased the body weight, adipose tissue mass and triglyceride and attenuated steatosis in liver. Moreover, the effect of oleuropein on adiponectin, an important hormone with fatty-acid oxidation properties, was evaluated and our data illustrated that oleuropein supplementation increased serum adiponectin concentration. The effects of oleuropein on protein expression related to lipogenic genes were investigated and our results showed that its administration significantly inhibited peroxisome proliferator-activated receptor $\gamma$ (PPAR $\gamma$ ), sterol regulatory element-binding protein-1c (SREBP-1c) and fatty-acid synthase (FAS). In addition, oleuropein stimulated the HCD-induced inhibition of AMP-activated protein kinase (AMPK) in epididymal adipose tissues. These results suggest that oleuropein exerts anti-obesity effects in HCD rats by activating AMPK and suppressing PPAR $\gamma$ (Peroxisome proliferator-activated receptor $\gamma$ ) expression in adipose tissues. These data provide that oleuropein has important implications for preventing obesity.
\end{abstract}

Keywords: Oleuropein; Adiponectin; AMPK; Fatty acid synthase; PPAR $\gamma$. 


\section{Introduction}

Obesity is a major risk factor for the development of various metabolic disorders including hyperlipidemia, type 2 diabetes, hypertension, cardiovascular disease and Nonalcoholic fatty liver disease (NAFLD) [1]. Obesity results from a prolonged positive energy imbalance needing to be reversed for treatment to be successful [2].

Adenosine monophosphate activated protein kinase (AMPK), a key enzyme of energy metabolism, regulates glucose and lipid uptake, storage and utilization in adipose tissue, liver and skeletal muscle [3-4]. AMPK is also involved in the regulation of peroxisome proliferator-activated receptor gamma (PPAR $\gamma)$ and CCAAT/enhancer-binding protein alpha $(\mathrm{C} / \mathrm{EBP} \alpha)$, which are the central regulators of adipogenesis [5]. Thus, agents that activate AMPK may reverse the effects of obesity related metabolic disorders such as type 2 diabetes and insulin resistance [6]. Several recent studies investigating the use of various medicinal plants to treat obesity, have been reported [7]. Hence, the development of AMPK activators has become a focus in the development of therapeutics for metabolic diseases such as diabetes and obesity [8].

Adipokines, hormones secreted from white tissue adipocyte (WAT), have been implicated in regulating insulin sensitivity. Adiponectin is an adipokine that acts as an insulin sensitizer, stimulating fatty acid oxidation in liver and muscle. Furthermore, adiponectin can be effective in the enhancement of the hepatomegaly, steatosis and alanine amino transferase abnormalities associated with NAFLD in mice [9].

Many different approaches to controlling obesity; such as drugs suppressing appetite or inhibit nutrient absorption have been suggested to date [10]. At present, clinically available anti-obesity drugs include Orlistat, an intestinal lipase inhibitor, and 
sibutramine, a centrally acting inhibitor of serotonin and norepinephrine reuptake [11]. However, these agents cause serious adverse effects, including insomnia, asthenia, constipation, emesis, headache, and stomachache [12]. Therefore, there is a demand for alternative therapies using herbal medicines with minimal side effects [8].

Olea europaea $L$. has been cited more than a thousand years and has been used widely in folk medicine in Mediterranean countries such as France, Greece, Italy, Morocco, Spain, Tunisia and Turkey. Olive leaves have been used in the human diet as extracts, herbal teas, and powder and contain several potentially bioactive compounds that may have antioxidant, antihypertensive, anti-atherogenic, anti-inflammatory, hypoglycemic, and hypocholesterolemic properties [13]. Oleuropein is the main phenolic compound of olive leaves, which is responsible for their pharmacological properties [14]. Several in vitro studies have demonstrated that oleuropein has a high antioxidant activity comparable to a hydrosoluble analog of tocopherol [15], and also inhibits the proliferation as well as the migration of various cancer cell lines, such as leukemia, melanoma, colon, breast, and kidney cancer cells [16].

It has been demonstrated in vivo that oleuropein decreased body weight gain [17], but oleuropein concentration used was strikingly different. In this context, Park et al. have demonstrated that oleuropein, at low concentration, attenuated hepatic steatosis induced by high-fat diet in mice [18]. More recent reports have shown that oleuropein supplementation at high concentration ( $0.59 \%)$ significantly decreased the body weight, decreased leptin concentration and modulated genes expression related to obesity in wild type male C57BL/6JOlaHsd mice [19]. However, the effect of oleuropein on adiponectin level and AMPK expression in obese rats were not established. Therefore, we 
hypothesized that supplementation of oleuropein would increase adiponectin level gain by modulating the expression level of genes associated with lipid metabolism essentially AMPK.

\section{Materials and Methods}

\subsection{Preparation of oleuropein}

Olea europaea leaves from chemlali cultivar were collected in the area of Sfax prefecture (South east of Tunisia). The species is a member of the Oleaceae family and is referenced in the latest revision of "The Plant list" under record key 371818. A specimen voucher was deposited at the herbarium of the LBP laboratory under the number LBP-OL-9. Oleuropein used in the present study was purified from olive leaves, dried under microwaves and powdered. $100 \mathrm{~g}$ of ground olive leaves were soaked in $500 \mathrm{ml}$ distilled water. The mixture was stirred at room temperature overnight, then paper filtered and the aqueous phase was extracted thrice with equal volume of ethyl acetate. After drying the organic phase under vacuum, the residue is chromatographed on silica gel at low pressure using a mixture of methylene chloride:methanol. Oleuropein structure was confirmed through the analysis of its UV spectrum and retention time in comparison to authentic standard obtained from Extrasynthese. The purified fraction was dried in a rotary evaporator, further lyophilized, and then stored until use. The purity of oleuropein was $97 \%$ with a purification yield of $5.7 \%$ of dry olive leaves. 


\subsection{Rats and diet}

Fifty male Wistar rats weighing between 150 and $170 \mathrm{~g}$ were purchased from Pasteur Institute (Tunis, Tunisia). The animals were maintained in individual stainless steel cages under controlled conditions $\left(23 \pm 1^{\circ} \mathrm{C}, 12 \mathrm{~h}\right.$ light-dark cycle, and had access to a standard laboratory diet (SICO, Sfax, Tunisia) and drinking water). The animals were housed according to the EEC 609/86 Directives regulating the welfare of experimental animals.

The rats were randomly divided into 3 experimental groups $(n=5)[20-21]$.

Group 1 was fed a standard laboratory diet (CD) (2900 kcal/kg). Group 2 was fed a cholesterol-rich diet (HCD) (3987.5 kcal/kg: normal diet supplemented with $10 \%$ cholesterol, $5 \%$ fructose and $0.1 \%$ bile salts). Groups 3 received HCD and daily gavaged with oleuropein (OL) $(50 \mathrm{mg} / \mathrm{kg})$. The experiment was conducted over a period of 8 weeks. The volume of the doses was kept constant at $1 \mathrm{~mL}$, and the normal control rats were administered $1 \mathrm{~mL}$ of distilled water. The body weight was measured every day. At the end of the experimental period, rats were sacrificed by decapitation and blood samples were collected to determine the plasma lipid profile. The livers and the adipose tissue were removed and rinsed with physiological saline solution. All samples were stored at $-80^{\circ} \mathrm{C}$ until analyzed.

\subsection{Serum biochemical parameter analysis}

Blood glucose levels were determined using a commercially available glucose kit (Biolabo, France) based on the glucose oxidase method. Creatinine, Aspartate aminotransferase (AST) and alanine aminotransferase (ALT) activities, serum lipid levels of triglycerides (TG), total cholesterol (TC), high density lipoprotein cholesterol (HDL-c) 
and low density lipoprotein cholesterol (LDL-c) were measured on an automatic biochemistry analyzer (Vitalab FlexorE, USA) at the clinical and Biochemical laboratory of the Hedi Chaker University Hospital, Sfax- Tunisia.

Serum adiponectin level was determined using adiponectin ELISA kit (Crystal Chem Inc., Chicago, IL, USA), according to the manufacturer's instructions. Serum samples were diluted in sample diluent (kit) for optimal fit within the calibration curve and were measured in duplicate.

\subsection{Histological analysis of white adipose tissue and liver}

At the end of the experimental period, the rats were sacrificed. After blood collection, the white adipose tissue and liver were removed from the rats and weighed immediately. For adipocyte staining, adipose tissues were fixed in $10 \%$ neutral formalin solution for 1 day and then embedded in paraffin. All tissues were cut to a thickness of $6 \mu \mathrm{m}$ and stained with hematoxylin and eosin. The stained sections were analyzed using light microscopy (Olympus BX51, Olympus Optical Co).

Semi-quantitative assessment of liver injury was performed using scores ranging from 0 to 4 as follows: representing a $25 \%$ loss $(1+)$, representing a $50 \%$ loss $(2+)$, representing a $75 \%$ loss $(3+)$ and representing more than $75 \%$ loss $(4+)$ [22-23]. The scores were determined in each section selected randomly and 20 fields were examined using $1000 \times$ magnification. 


\subsection{Western Blot}

Adipose tissues of each rat were pooled and homogenized at $4{ }^{\circ} \mathrm{C}$ in RIPA buffer. The tissue homogenates were centrifuged $\left(1300 \mathrm{~g}, 20 \mathrm{~min}, 4{ }^{\circ} \mathrm{C}\right)$ and the resulting supernatants (whole-tissue extracts) were used for the Western blot analysis. The total protein concentrations of the whole-tissue extracts were determined by Bradford assay (Bio-Rad, CA, USA). Protein samples were separated with 12\% SDS-PAGE, transferred onto a nitrocellulose membrane (Amersham, Buckinghamshire, UK) and hybridized with primary antibodies (diluted 1:1000), overnight at $4{ }^{\circ} \mathrm{C}$. Antibodies for peroxisome proliferator-activated receptor $\gamma(\operatorname{PPAR} \gamma)$, SREBP-1c (sterol regulatory element-binding protein-1c) and $\beta$-actin detection were purchased from Santa Cruz Biotechnology (Santa Cruz, CA, USA), and antibodies for fatty-acid synthase (FAS), AMPK $\alpha$, and phosphoAMPK $\alpha$ were purchased from Cell Signaling Technology (Beverly, MA, USA). After incubation with primary antibodies, the membranes were incubated with the corresponding horseradish peroxidase-conjugated secondary antibodies (Promega) for $1 \mathrm{~h}$ at room temperature. The membranes were then treated with the ECL detection reagent (Amersham Bioscience, England), and protein bands were visualized using Las-1000 (Fujifilm, Tokyo, Japan). The relative band density was determined by densitometry with Image J software (National Institutes of Health, Bethesda, MD, USA).

\subsection{Statistical analysis}

Statistical analysis was performed using SPSS system version 19. All data are presented as means $\pm \mathrm{SD}$ for each group of animals. The results from each experimental group were 
compared using one-way analysis of variance followed by the Tukey's post hoc test. P $<0.05$ was considered statistically significant.

\section{Results}

3.1. Body weight and food intake

Oleuropein significantly prevented $(\mathrm{p}<0.05)$ the increase of the rat body weight compared to the control (Table. 1). The increase in body weight in oleuropein treated group was 92.4 g compared to the $145.28 \mathrm{~g}$ increase in the HCD group. However, no significant food intake (19-20 g/day) difference was observed among experimental groups. After 60 days, the epididymal white adipose tissue (EpiWAT) weight was significantly decreased by oleuropein treatment.

\subsection{Effects of oleuropein on serum metabolic parameters}

The triacylglycerols, total cholesterol, and LDL cholesterol serum concentrations were higher in rats fed with the HCD diet compared to those given a standard diet. (Table 2). The oleuropein supplementation significantly $(\mathrm{p}<0.05)$ reduced plasma concentration of TG, TC and LDL-C and increased HDL-C.

\subsection{Effect of oleuropein on adiponectin secretion}

To examine whether oleuropein modulated adiponectin production, we measured adiponectin level in serum levels. Compared with the control group, the HCD group had lower levels of adiponectin in serum. However, administration of oleuropein significantly increased serum levels of this hormone (Fig. 1). 


\subsection{Effects of oleuropein on adipose tissue and hepatic steatosis}

The weight of the epididymal white adipose tissue in the HCD group was higher than those in the control (Fig. 2A). The oleuropein treatment significantly decreased $(p<0.05)$ all of the white adipose tissue weights. The histological examinations revealed that the adipocyte size in the epididymal adipose tissue was larger in the HCD group than in that of the control group (Fig. 2B). The oleuropein treatment significantly $(\mathrm{p}<0.05)$ decreased the adipocytes size which were similar to that in control group (Fig. 2C).

The effect of oleuropein administration on steatosis was also elucidated and the results showed that liver weight was significantly lower in $\mathrm{HCD}+\mathrm{OL}$ group in comparison to the HCD group (Fig. 3A). The histological analysis showed a significant increase in lipid deposition in the livers of the HCD rats (Fig. 3B), characteristic of accentuated microand macro-vesicular steatosis, accompanied by an inflammatory cell infiltration. A noticeable increase in the steatosis score was seen in livers of the HCD group, as compared to the control group (Fig. 3C). These histological data were consistent with the reduced hepatic TG content and ALT serum levels in the $\mathrm{HCD}+\mathrm{OL}$ rats group, indicating that oleuropein improved hepatic morphology and its function (Table 3).

\subsection{Molecular mechanism of lipids metabolism control}

To elucidate the mechanism underlying the effects of oleuropein on lipid metabolism, we evaluated the protein expression levels of various genes involved in lipogenesis and lipolysis, in epididymal adipose tissue (Fig. 4A). Compared to the control (untreated) group, the HCD group exhibited an increase in protein levels of adipocyte markers such as PPAR $\gamma$, SREBP-1c and FAS. The levels of these proteins were significantly lower in 
the HCD supplemented with oleuropein. The effect of oleuropein on AMPK was also investigated and the results showed that the AMPK phosphorylation in the HCD + OL group has increased compared to that in the HCD group (Fig. 4A and B).

\section{Discussion}

This study revealed the regulatory mechanism of oleuropein underlying its anti-obesity effects in HCD rats especially the regulation of adiponectin and the signaling of AMPK. Our findings showed that oleuropein is effective in the prevention of body weight increased in HCD fed rats. Indeed, the weight gain in the last group was highly noticeable in comparison with the control rats. However, oleuropein administration resulted in a significant body weight reduction. Excessive growth of adipose tissue resulting in obesity includes two growth mechanisms: hyperplasia (increase in number of cells) and hypertrophy (increase in cell size). Our results showed that Chronic HCD feeding resulted in increasing adipocyte size. The histological appearance of WAT in the HCD group supplemented with oleuropein was more regular and showed adipocyte size similar to that of the control group. In addition, the protective effect of oleuropein against hepatocyte stress and steatosis was also evaluated. The steatosis score, the AST and ALT activities have been attenuated in the presence of oleuropein compared to HCD.

The white adipose tissue is the major energy reservoir in mammals and plays a key role in the maintenance of energy homeostasis [24]. PPAR is a key transcription factor in adipocyte differentiation, lipid storage, and glucose homeostasis [25] and it regulates the expression of adipocyte markers such as SREBP-1c,. FAS and aP2. SREBP-1 is the most important transcription factor regulating de novo lipogenesis in the liver and is 
responsible primarily for the regulation of genes involved in fatty acid biosynthesis, including FAS [26]. It is known that SREBP-1c is negatively regulated by AMPK [27]. AMPK is a promising target for the treatment of metabolic disorders such as obesity and type-2 diabetes. Once activated, AMPK exerts an anti-lipogenic effect by inhibiting glucose and fatty-acid uptake in adipocytes [24]. AMPK activate mitochondrial fatty-acid oxidation for lipid utilization and modulates adipocyte lipolysis [3]. In our study, we found that HCD feeding significantly increased SREBP-1c and FAS expression in epididymal WAT of rats, in parallel with attenuated AMPK phosphorylation, indicating that the negative feedback regulation of SREBP-1c via AMPK failed in HCDoverloading conditions. Additionally, we found that oleuropein significantly suppressed PPAR $\gamma$, SREBP-1c and FAS expression by promoting AMPK phosphorylation.

Our findings are in agreement with the report of Son et al. who have demonstrated that flavonoids and polyphenols are able to suppress adipocyte differentiation by activating AMPK [28]. Also, Hao et al. showed in vitro that hydroxytyrosol activated AMPK in adipocytes and muscle cells, after 30 min of exposure [29]. Furthermore, adiponectin, a protein of 244 amino acid, is active and highly expressed in adipocytes [30]. This protein increased insulin sensitivity by stimulating fatty acid oxidation, decreases plasma triglycerides, improves glucose metabolism and is able to activate AMPK in adipose tissues [31]. Adiponectin has recently attracted much attention because its decrease in the circulating levels causes the development of obesity, insulin resistance, type 2 diabetes and atherosclerosis. For this reason, adiponectin is regarded as being a crucial tool for the regulation of obesity [32]. Administration of oleuropein was associated with increased circulating adiponectin levels. Thus, oleuropein may reduce lipid levels by 
suppressing lipid synthesis and promoting fatty-acid oxidation via the regulation of AMPK activation. Previous reports have demonstrated that Pioglitazone, a high-affinity ligand for PPAR $\gamma$; improves insulin resistance in diabetics but often leads to weight gain [33]. However, the use of pioglitazone is associated with an increased risk of incident bladder cancer among people with type - 2 diabetes [34]. When compared to pioglitazone [35], we found that oleuropein has comparable effects. Nevertheless, further studies are still needed to clarify the role of PPAR $\gamma$ in the hypolipidemic effect of oleuropein. Moreover, more researchers have investigated the effect of oleuropein on cardiovascular disease via AMPK activation [15-36]. We should note that this is the first study dealing with the use of oleuropein at low concentration to prevent obesity in high cholesterol diet fed rats, by activating AMPK and enhancing adiponectin production. Several recent reports have shown that administration of oleuropein at high concentration $(0.59 \% \mathrm{w} / \mathrm{w})$ showed an improved health status compared to the control HFD-fed mice and significantly decreased the body weight gain, body fat accumulation, leptin level and plasma TG concentrations in mice [19]. In another report, Park et al. have demonstrated that oral administration of oleuropein $(0.03 \% \mathrm{w} / \mathrm{w})$ attenuated hepatic steatosis induced by high-fat diet in mice. Indeed, oleuropein regulated the hepatic expression of adipogenic nuclear-transcription factors (PPARc2 and LXR) and their target genes (LPL and aP2). Oleuropein-supplemented HFD resulted also in decreased mRNA levels of FGF1-mediated signaling molecules (FGFR1, CycD, andE2F1) and hepatic ERK phosphorylation in of experimental mice [18]. Based on these studies, the prevention of HFD induced body weight gain was dependent on oleuropein concentration. In fact, Ebaid et al., demonstrated that oleuropein administration at $0.023 \mathrm{mg} / \mathrm{kg}$ (via gavage) did 
not prevent HFD induced body weight gain in rats [37]. However, a preventive effect of oleuropein was observed when the later was used at $30 \mathrm{mg} / \mathrm{kg}$, as previously shown by Park et al. [18]. Concerning the food intake, we showed that oleuropein did not affect energy intake. Thus, we could hypothesize that visceral fat-pad weight lowering effect of oleuropein could be mediated by inhibition of adipogenesis without affecting cumulative energy intake. Our results are in agreement with those of park et al., who also demonstrated that oleuropein administration $(0.03 \%)$ did not affect the energy intake. Recently, Ying et al., proved that olive leaves extract contained $0.024 \%$ significantly reduced food intake, whereas oleuropein alone did not affect food intake in mice [18-38]. These results confirmed that oleuropein at low concentration has no effect on energy intake. At high concentration of oleuropein $(0,59 \%)$, Vander stelt et al. showed that the use of high dose of oleuropein involved a short-term mechanism affecting the intestine and consequently the energy uptake and a long-term homeostatic adaptation to this, resulting in a higher satiety of the mice [19].

In summary, the oleuropein administration in HCD rats reduced body weight gain, adipose mass and serum triglyceride concentration. These results suggest that oleuropein exerts anti-obesity effects in HCD-induced obese rat by modulating lipids metabolism by down-regulating the expression of PPAR $\gamma$, increasing adiponectin levels in serum and activating AMPK in white adipose tissue. The anti-obesity effects of oleuropein in HCD rats support the potential use of oleuropein as a therapeutic agent for reducing the risks of various metabolic diseases. 


\section{Conflict of interest}

The authors declare that there have no conflicts of interest.

\section{Acknowledgment}

This research was partially supported by the JICA-JST Science and Technology Research Partnership for Sustainable Development (SATREPS) Project: "Valorization of BioResources in Semi-Arid and Arid Land for Regional Development".

\section{References}

[1] B. Jiang, Y. Liang, X. Sun, X. Liu, W. Tian, X. Ma, Potent Inhibitory Effect of Chinese Dietary Spices on Fatty Acid Synthase, Plant foods Hum Nutr.70 (2015) 257-262.

[2] J.S. You, Y.J. Lee, K.S.Kim, S.H.Kim, K.J. Chang, Ethanol extract of lotus (Nelumbo nucifera) root exhibits an anti-adipogenic effect in human preadipocytes and anti-obesity and anti-oxidant effects in rats fed a high-fat diet, Nutr Res. 34 (2014) 258- 267.

[3] S.K. Kim, C.-S. Kong, Hwang, Anti-adipogenic effect of dioxinodehydroeckol via AMPK activation in 3T3-L1 adipocytes, Chem. Biol Interact. 186 (2010) 24-29.

[4] M.S. Seo, S.W. Hong, S.H. Yeon, Y.M. Kim, K.A. Um, J.H. Kim, H.J. Kim, K.C. Chang, S.W. Park, Magnolia officinalis attenuates free fatty acid- 427 induced lipogenesis via AMPK phosphorylation in hepatocytes, J. Ethnopharmacol. 157 (2014) 140-148.

[5] B. Zou, Z. Ge, Y. Zhang, J. Du, Z. Xu, C. Li, Persimmon tannin accounts for hypolipidemic effects of persimmon through activating of AMPK and suppressing NF- $\kappa \mathrm{B}$ activation and inflammatory responses in high-fat diet rats, Food Funct. 5 (2014) 1536-1546.

[6] Y. Yamashita, L. Wang, L. Wang, Y. Tanaka, T. Zhang, H. Ashida, Oolong, black and pu-erh tea suppresses adiposity in mice via activation of AMP-activated protein kinase, Food Funct. 5 (2014) 2420-2429.

[7] H. Choi, H. Eo, K. Park, M. Jin, E.-J. Park, S.-H. Kim, A water-soluble extract from Cucurbita moschata shows anti-obesity effects by controlling lipid metabolism in a high fat diet-induced obesity mouse model, Biochem. Biophys. Res. Commun. 359 (2007) 419-425. 
[8] B.B. Kahn, T. Alquier, D. Carling, D.G. Hardie, AMP-activated protein kinase: ancient energy gauge provides clues to modern understanding of metabolism, Cell Metab. 1 (2005) 15-25.

[9] D.G. Hardie, AMP-activated protein kinase: an energy sensor that regulates all aspects of cell function, Genes Dev. 25 (2011) 1895-1908.

[10] Y.-S. Lee, B.-Y. Cha, K. Saito, S.-S. Choi, X.X. Wang, B.-K. Choi, et al., Effects of a Citrus depressa Hayata (shiikuwasa) extract on obesity in high-fat dietinduced obese mice, Phytomedicine. 18 (2011) 648-654.

[11] B. Sharma, D.C. Henderson, Sibutramine: current status as an anti-obesity drug and its future perspectives, Expert Opin. Pharmacother. 9 (2008) 2161-2173.

[12] P. Bansal, P. Paul, J. Mudgal, P.G. Nayak, S.T. Pannakal, K.I. Priyadarsini, M.K. Unnikrishnan, Antidiabetic, antihyperlipidemic and antioxidant effectsof the flavonoid rich fraction of Pilea microphylla (L.) in high fat diet/streptozotocininduced diabetes in mice, Exp. Toxicol. Pathol. 64 (2012) 651-658.

[13] A. Kaeidi, S. Esmaeili-Mahani, V. Sheibani, M. Abbasnejad, B.Rasoulian, Z.Hajializadeh, S.Afrazi, Olive (Olea europaea L.) leaf extract attenuates early diabetic neuropathic pain through prevention of high glucose-induced apoptosis: In vitro and in vivo studies, J. Ethnopharmacol. 136 (2011) 188-196.

[14] J. Del Río, Enhancement of phenolic compounds in olive plants (Olea europaea L.) and their influence on resistance against Phytophthora sp., Food Chem. 83 (2003) 75-78.

[15] I. Andreadou, E.K. Iliodromitis, E. Mikros, M. Constantinou, A. Agalias, P. Magiatis, et al., The olive constituent oleuropein exhibits anti-ischemic, antioxidative, and hypolipidemic effects in anesthetized rabbits, J. Nutr. 136 (2006) 2213-2219.

[16] J. Han, T.P.N. Talorete, P. Yamada, H. Isoda, Anti-proliferative and apoptotic effects of oleuropein and hydroxytyrosol on human breast cancer MCF-7 cells., Cytotechnology. 59 (2009) 45-53.

[17] H. karim, I. Hassen, H. Casabinava, Biological activities of the natural antioxidant oleuropein: Exceeding the expectation - A mini-review, J. Funct. Foods. 18 (2015) 926-940.

[18] S. Park, Y. Choi, S.-J. Um, S.K. Yoon, T. Park, Oleuropein attenuates hepatic steatosis induced by high-fat diet in mice, J. Hepatol. 54 (2011) 984-93. 
[19] I. van der Stelt, E.F. Hoek-van den Hil, H.J.M. Swarts, J.J.M. Vervoort, L. Hoving, L. Skaltsounis, et al., Nutraceutical oleuropein supplementation prevents high fat diet-induced adiposity in mice, J. Funct. Foods. 14 (2015) 702-715.

[20] H. Ben Ayed, R. Nasri, N. Jemil, I. Ben Amor, J. Gargouri, N. Hmidet,M. Nasri, Acute and sub-chronic oral toxicity profiles of lipopeptides from Bacillus mojavensis A21 and evaluation of their in vitroanticoagulant activity, Chem. Biol Inter. 236 (2015) 1-6.

[21] R. Nasri, O. Abdelhedi, I. Jemil, I. Daoued, K. Hamden, C. Kallel, A. Elfeki, M. Lamri-Senhadji, A. Boualga, M. Nasri, M. Châabouni, Ameliorating effects of goby fish protein hydrolysates on high-fat-high-fructose diet-induced hyperglycemia, oxidative stress and deterioration of kidney function in rats, Chem. Biol. inter. 242 (2015) 71-82.

[22] T.N. Slaughter, A. Paige, D. Spires, N. Kojima, P.B. Kyle, M.R. Garrett, Characterization of the development of renal injury in Type-1 diabetic Dahl saltsensitive rats, Am J Physiol Regul Integr Comp Physiol. 305 (2013) 727-734.

[23] A. Mahmoudi, H. Ghorbel, Z. Bouallegui, R. Marrakchi, H. Isoda, S. Sayadi, Oleuropein and hydroxytyrosol protect from bisphenol A effects in livers and kidneys of lactating mother rats and their pups', Exper. Toxicol Pathol. 67 (2015) 413-425

[24] S.-J. Kim, J.Y. Jung, H.W. Kim, T. Park, Anti-obesity effects of Juniperus chinensis extract are associated with increased AMP-activated protein kinase expression and phosphorylation in the visceral adipose tissue of rats, Biol. Pharm. Bull. 31 (2008) 1415-1421.

[25] W.-X. Liu, T. Wang, F. Zhou, Y. Wang, J.-W. Xing, S. Zhang, et al., Voluntary exercise prevents colonic inflammation in high-fat diet-induced obese mice by upregulating PPAR- $\gamma$ activity, Biochem. Biophys. Res. Commun. 459 (2015) 47580 .

[26] R.M. Hagen, S. Rodriguez-Cuenca, A. Vidal-Puig, An allostatic control of membrane lipid composition by SREBP1, FEBS Lett. 584 (2010) 2689-98.

[27] Y. Li, S. Xu, M.M. Mihaylova, B. Zheng, X. Hou, B. Jiang, et al., AMPK phosphorylates and inhibits SREBP activity to attenuate hepatic steatosis and atherosclerosis in diet-induced insulin-resistant mice, Cell Metab. 13 (2011) 37688 .

[28] Y. Son, J.-S. Nam, M.-K. Jang, I.-A. Jung, S.-I. Cho, M.-H. Jung, Anti-obesity Activity of Vigna nakashimae Extract in High-Fat Diet-Induced Obesity, Biosci Biotechnol. Biochem. 77(2014) 332-338. 
[29] J. Hao, W. Shen, G. Yu, H. Jia, X. Li, Z. Feng, et al., Hydroxytyrosol promotes mitochondrial biogenesis and mitochondrial function in 3T3-L1 adipocytes, J. Nutr. Biochem. 21 (2010) 634-44.

[30] T.-S. Tsao, H.F. Lodish, J. Fruebis, ACRP30, a new hormone controlling fat and glucose metabolism, Eur. J. Pharmacol. 440 (2002) 213-221.

[31] C.-H. Wu, S.-C. Chen, T.-T. Ou, C.-C. Chyau, Y.-C. Chang, C.-J. Wang, Mulberry leaf polyphenol extracts reduced hepatic lipid accumulation involving regulation of adenosine monophosphate activated protein kinase and lipogenic enzymes, J. Funct. Foods. 5 (2013) 1620-1632.

[32] M.-J. Lee, Y.K. Rao, K. Chen, Y.-C. Lee, Y.-M. Tzeng, Effect of flavonol glycosides from Cinnamomum osmophloeum leaves on adiponectin secretion and phosphorylation of insulin receptor-beta in 3T3-L1 adipocytes, J. Ethnopharmacol. 126 (2009) 79-85.

[33] R.F. Kushner, M. Sujak, Prevention of weight gain in adult patients with type 2 diabetes treated with pioglitazone., Obesity (Silver Spring). 17 (2009) 1017-1022.

[34] L. Azoulay, H. Yin, K.B. Filion, J. Assayag, A. Majdan, M.N. Pollak, et al., The use of pioglitazone and the risk of bladder cancer in people with type 2 diabetes: nested case-control study, BMJ. 344 (2012) e3645.

[35] T.-F. Tzeng, H.-J. Lu, S.-S. Liou, C.J. Chang, I.-M. Liu, Reduction of lipid accumulation in white adipose tissues by Cassia tora (Leguminosae) seed extract is associated with AMPK activation, Food Chem. 136 (2013) 1086-1094.

[36] I. Andreadou, M. Papaefthimiou, A. Zira, M. Constantinou, F. Sigala, A.L. Skaltsounis, A. Tsantili-Kakoulidou, E.K. Iliodromitis, D.T. Kremastinos, E. Mikros, Metabonomic identification of novel biomarkers in doxorubicin cardiotoxicity and protective effect of the natural antioxidant oleuropein, NMR in Biomed. 22 (2009) 585-92

[37] G. M. Ebaid, F. R. Seiva, K. K. Rocha, G. A. Souza, E. L. Novelli, Effects of olive oil and its minor phenolic constituents on obesity-induced cardiac metabolic changes, J. Nutr. 9 (2010). 46

[38] Shen, S.J. Song, N. Keum, T. Park, Olive leaf extract attenuates obesity in highfat diet-fed mice by modulating the expression of molecules involved in adipogenesis and thermogenesis. Evid. Based Complement. Altern. Med. 2014, (2014), 971890. 


\section{Figures caption}

Fig. 1. Adiponectin concentration in serum. Values are expressed as the mean $\pm \mathrm{SD}$ $(n=5) . C D=$ normal fat diet, $\mathrm{HCD}=$ high cholesterol diet; $\mathrm{HCD}+\mathrm{OL}=$ high cholesterol diet supplemented with $50 \mathrm{mg} / \mathrm{kg}$ oleuropein. Significant differences were observed between the $\mathrm{C}$ and HCD groups: \#\#p $<0.01$. Significant differences were observed between the HCD and the HCD + OL groups: **p $<0.01$.

Fig. 2. Analysis of the adipose tissue. $C D=$ normal fat diet; $H C D=$ high cholesterol diet; $\mathrm{HCD}+\mathrm{OL}=$ high cholesterol diet supplemented with $50 \mathrm{mg} / \mathrm{kg}$ oleuropein. Significant differences were observed between the $\mathrm{C}$ and HCD groups: \#\#p $<0.01$. Significant differences were observed between the HCD and the HCD + OL groups: **p $<0.01$. (A) Weight of the epididymal white adipose tissue (epiWAT) after dissection, relative to body weight; (B) Histology of the epididymal adipose tissue stained with hematoxylin and eosin (magnification, 400×); (C) Measured adipocyte area of sections $(n=5)$.

Fig. 3. Study of the hepatic tissue. CD, normal fat diet; HCD, High cholesterol diet; $\mathrm{HCD}+\mathrm{OL}$ High cholesterol diet $+50 \mathrm{mg} / \mathrm{kg}$ oleuropein. Significant differences were observed between the $\mathrm{C}$ and HCD groups: \#\# $<0.01$. Significant differences were observed between the HCD and the HCD + OL groups: **p $<0.01$. (A) Liver weight; (B) Histological analysis of liver stained with hematoxylin and eosin (magnification, 400×); (C) Liver steatosis score. 
Fig. 4. Effect of oleuropein on SREBP-1C, FAS and PPAR $\gamma$ protein levels the phosphorylation of AMPK in epididymal adipose tissues. Representative bands (A) and Relative changes in protein expression levels (B). The relative expression levels of proteins were normalized against a $\beta$-actin internal control. The target-protein phosphorylation was normalized to the total protein-expression level. Values are expressed as means $\pm \mathrm{SD}(\mathrm{n}=5) . \mathrm{CD}=$ normal fat diet; $\mathrm{HCD}=$ High cholesterol diet; $\mathrm{HCD}+\mathrm{OL}=$ High cholesterol diet $+50 \mathrm{mg} / \mathrm{kg}$ oleuropein. Significant differences were observed between the $\mathrm{C}$ and HCD groups: $\# \mathrm{p}<0.05$. Significant differences were observed between the HCD and the HCD + OL groups: **p $<0.01$. 
Fig. 1

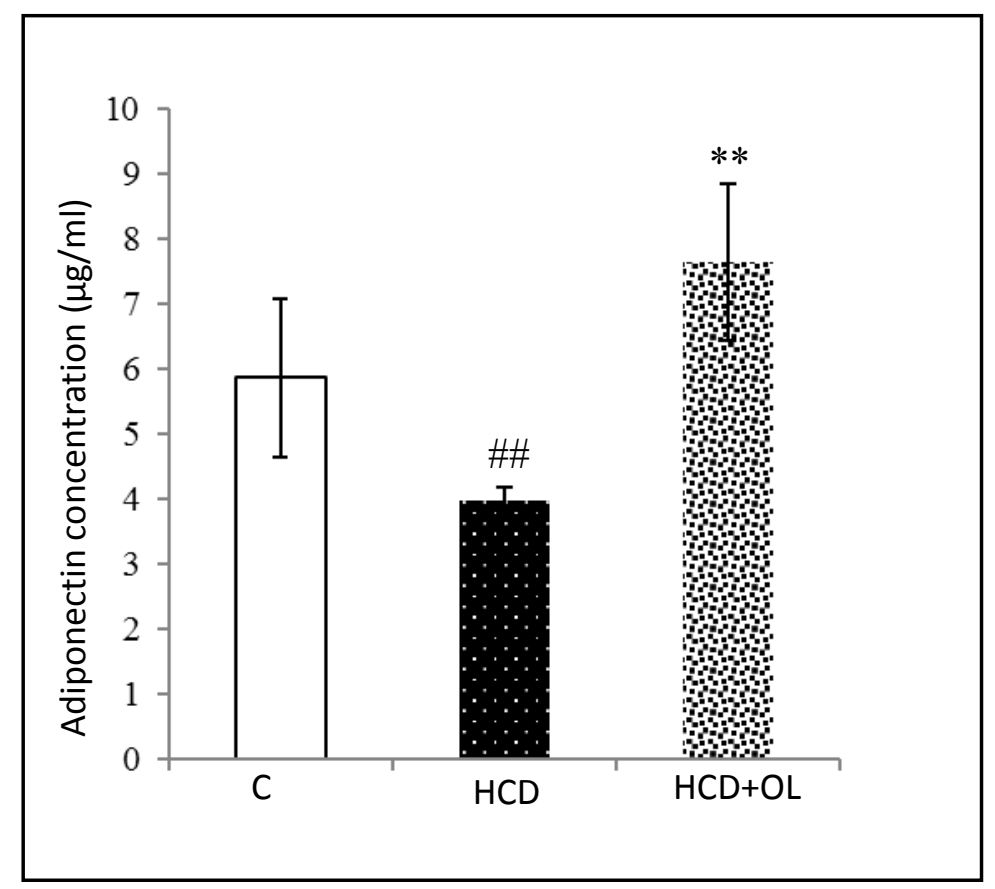


Fig. 2

(A)

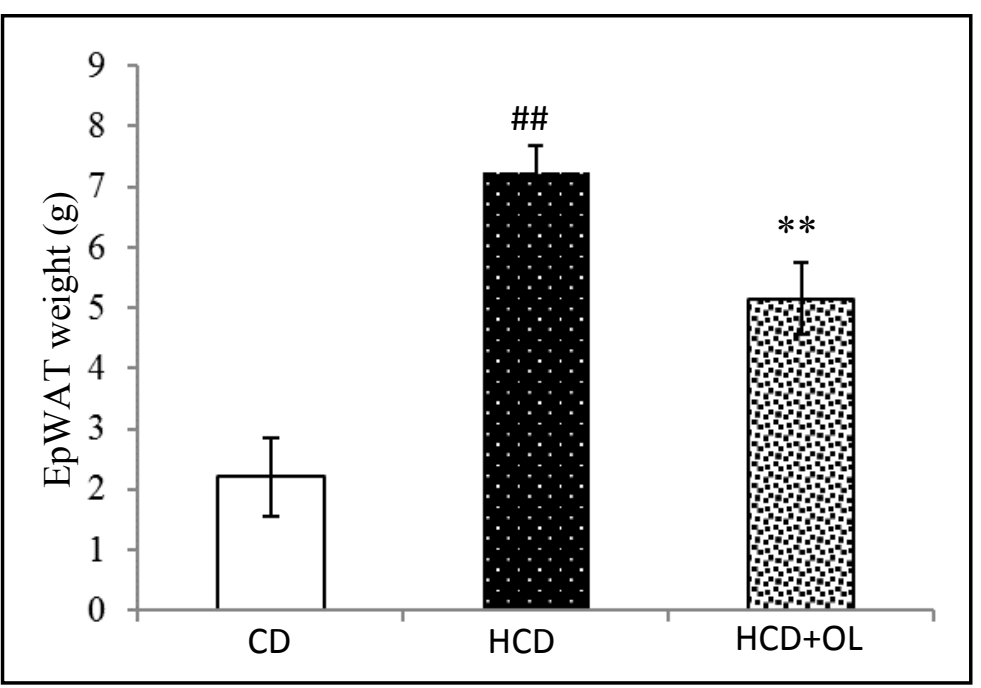

(B)

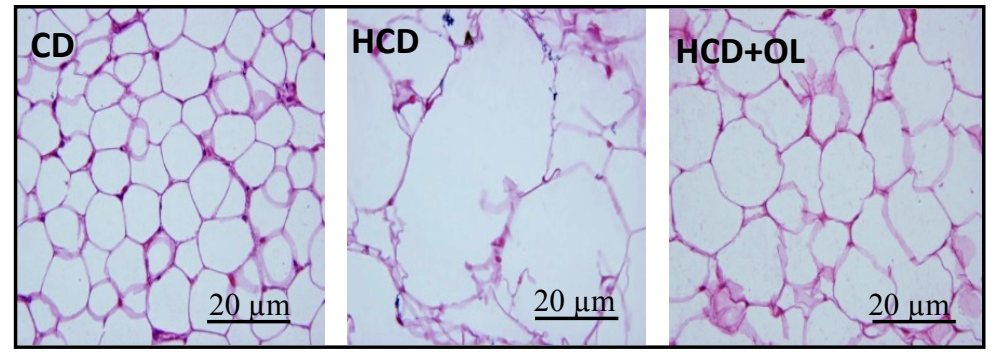

(C)

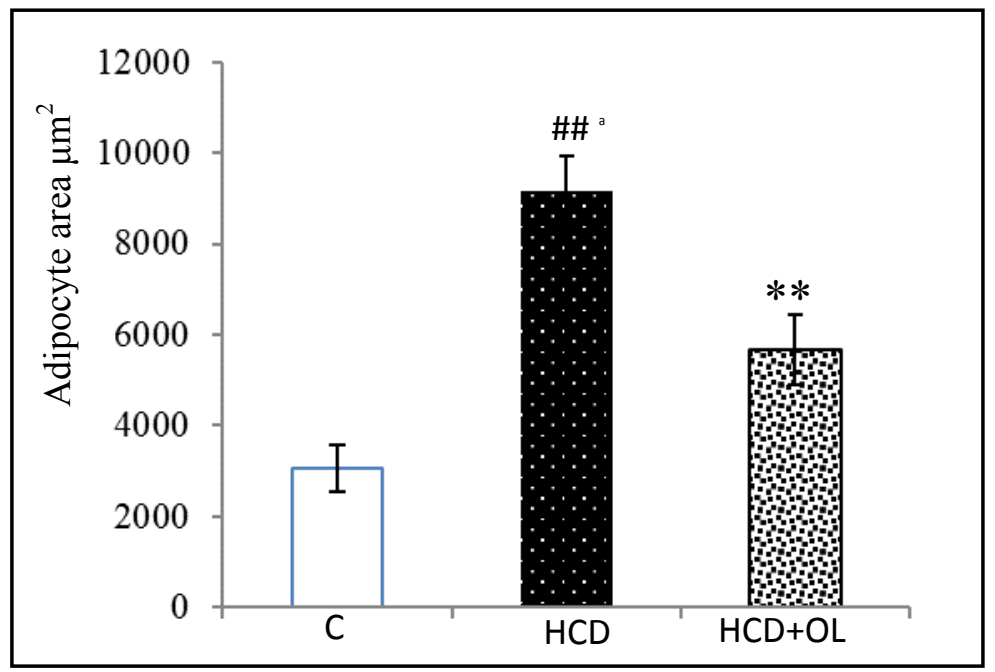


Fig. 3

(A)

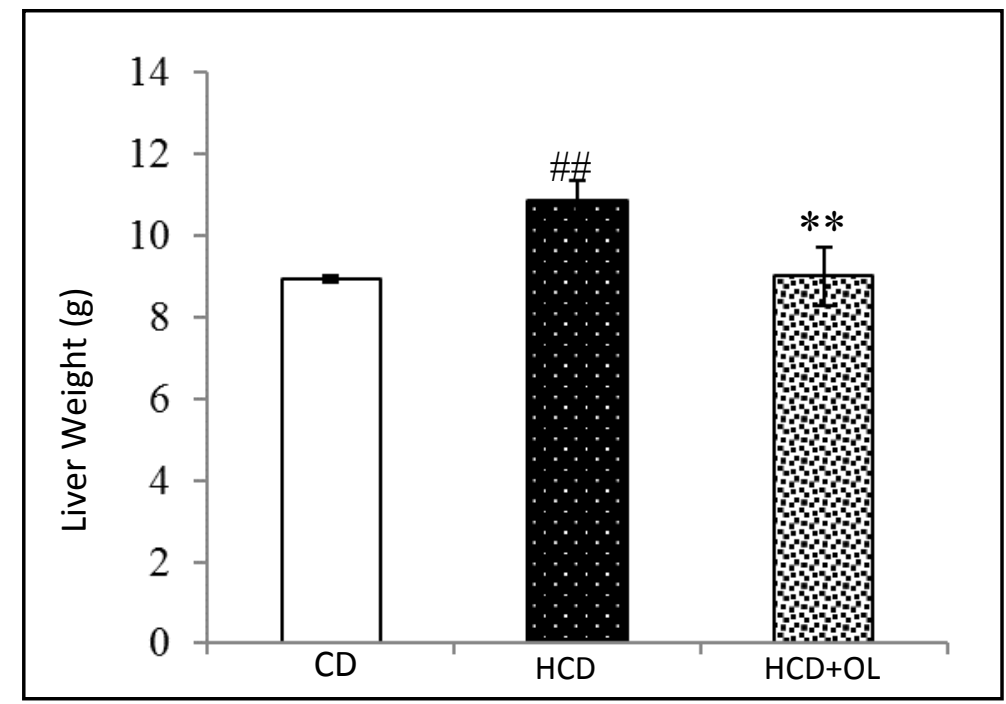

(B)

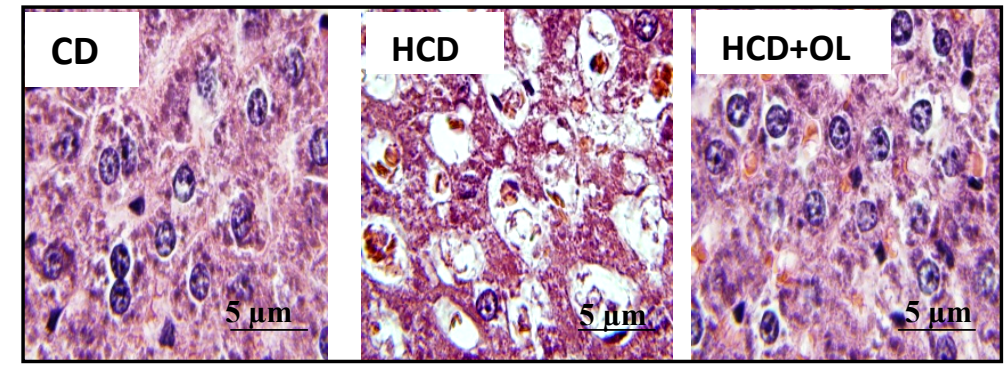

(C)

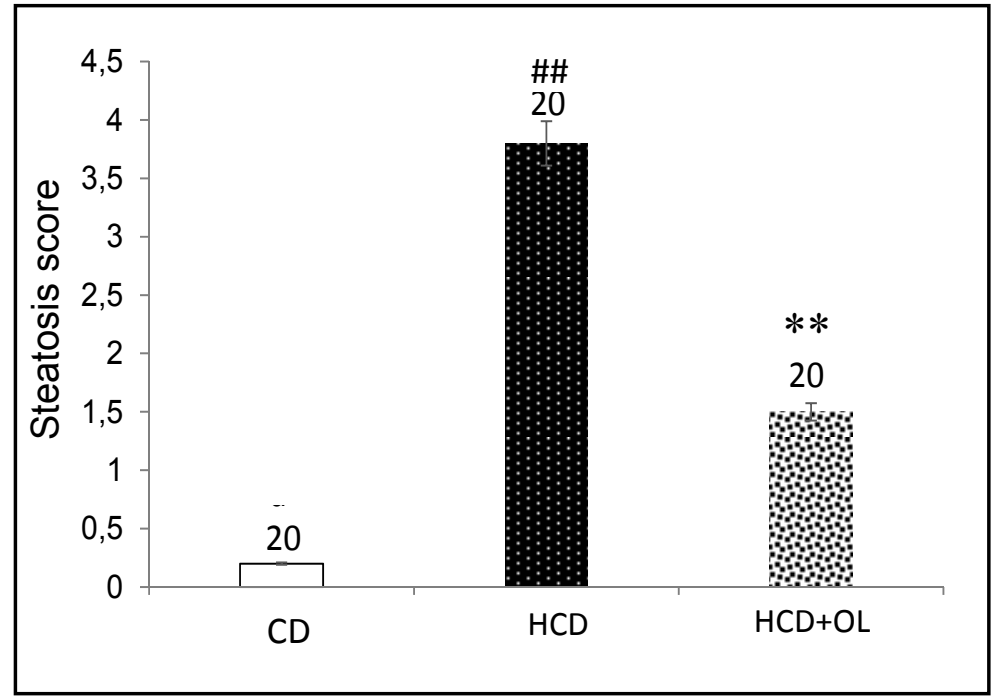


Fig. 4

(A)

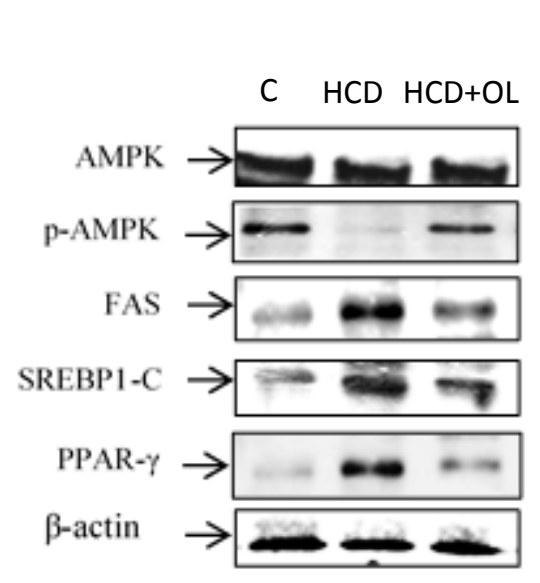

(B)

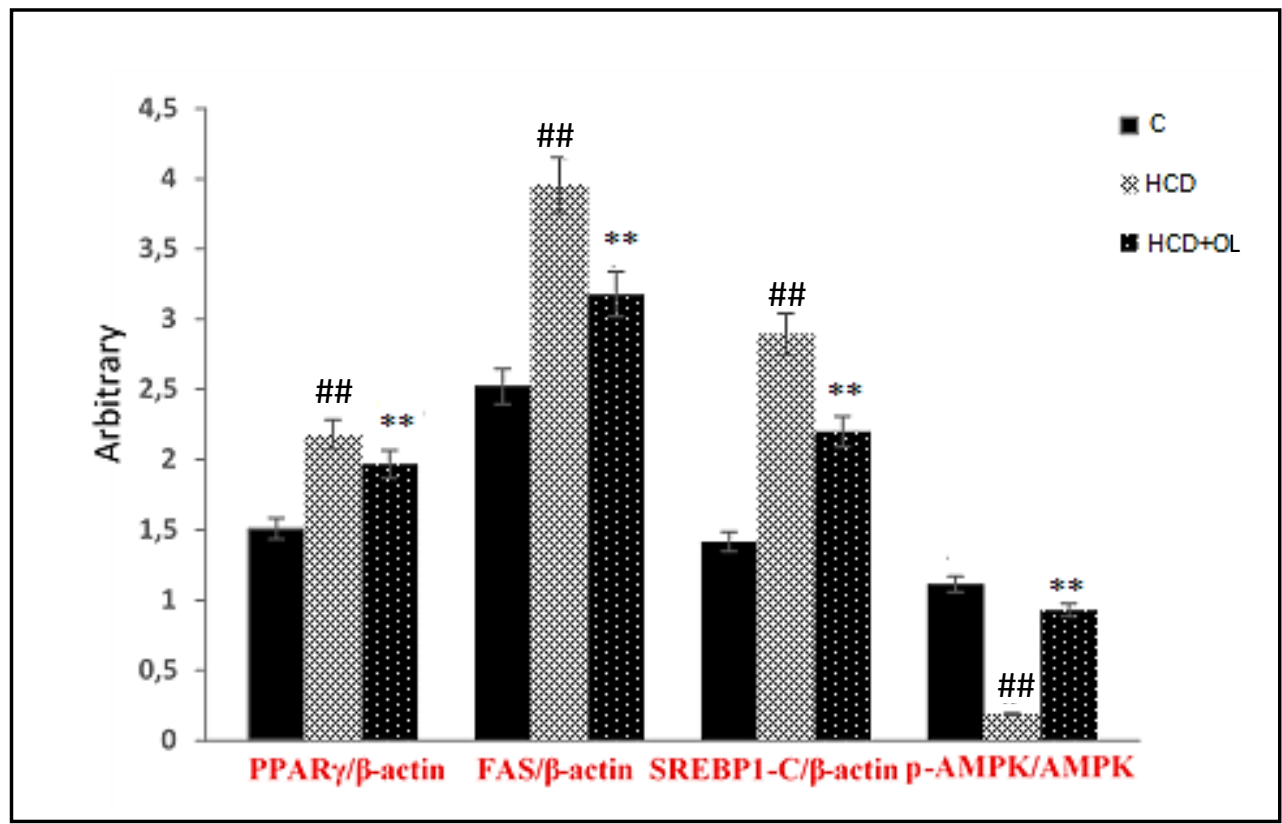


Table 1: Body weight, dietary intake and adipose tissue weights

\begin{tabular}{llll}
\hline & CD & HFD & HFD + OL \\
\hline Initial body weight $(\mathrm{g})$ & $144.6 \pm 5.98$ & $145.4 \pm 9.2$ & $144.4 \pm 7.2$ \\
Final body weight $(\mathrm{g})$ & $190.24 \pm 11.6$ & $290.68 \pm 10.05 \# \#$ & $237.37 \pm 10.17^{* *}$ \\
Food intake (g/day/rat) & $17.37 \pm 2.02$ & $21.044 \pm 4.04$ & $19.16 \pm 1.89$ \\
Calories intake (kcal/day) & $56.17 \pm 4.56$ & $79.11 \pm 4.09$ & $75.65 \pm 6.76$ \\
\hline
\end{tabular}

CD: rats fed a normal diet, HFD: rats fed high-fat diet, HFD+OL: rats fed high-fat diet with oleuropein $(50 \mathrm{mg} / \mathrm{kg})$. Values are presented as means standard deviations $(\mathrm{n}=5)$. "Significant differences were observed between the $\mathrm{C}$ and HCD groups: \#\# $<0.01$. Significant differences were observed between the HCD and the HCD + OL groups: **p $<0.01$ ". 
Table 2: Effect of oleuropein on serum lipid profiles in high-fat diet rats

\begin{tabular}{llll}
\hline & \multicolumn{2}{c}{ Groups } \\
\cline { 2 - 4 } Serum $(\mathrm{mmol} / \mathrm{L})$ & $\mathrm{CD}$ & HFD & HFD+OL \\
\hline TC & $0.88 \pm 0.23$ & $2.73 \pm 0.15 \# \#$ & $1.18 \pm 0.06^{* *}$ \\
TG & $1.08 \pm 0.19$ & $1.56 \pm 0.10 \# \#$ & $1.28 \pm 0.09^{* *}$ \\
LDL-C & $0.61 \pm 0.08$ & $0.91 \pm 0.07 \#$ & $0.65 \pm 0.03$ \\
HDL-C & $0.49 \pm 0.06$ & $0.35 \pm 0.03 \#$ & $0.41 \pm 0.10^{*}$ \\
\hline
\end{tabular}

CD: rats fed a normal diet, HFD: rats fed high-fat diet, HFD+OL: rats fed high-fat diet with oleuropein $(50 \mathrm{mg} / \mathrm{kg})$. Values are expressed as means $\pm \mathrm{SD}(\mathrm{n}=5)$. "Significant differences were observed between the $\mathrm{C}$ and HCD groups: $\# \mathrm{p}<0.05$; \#\# $\mathrm{p}<0.01$. Significant differences were observed between the HCD and the HCD + OL groups: ${ }^{*} \mathrm{p}<0.05 ;{ }^{* *} \mathrm{p}<0.01$. 
Table 3: Serum activities of alanine aminotransferase and aspartate aminotransferase in different rat groups

\begin{tabular}{|c|c|c|c|}
\hline & $\mathrm{CD}$ & HFD & $\mathrm{HFD}+\mathrm{OL}$ \\
\hline AST (UI/L) & $73 \pm 4.37$ & $95.4 \pm 3.83 \# \#$ & $83.2 \pm 4.2 * *$ \\
\hline $\operatorname{ALT}(\mathrm{UI} / \mathrm{L})$ & $38.7 \pm 2.05$ & $52.5 \pm 1.6 \# \#$ & $45.2 \pm 3.7 *$ \\
\hline Creatinine $(\mu \mathrm{mol} / \mathrm{L})$ & $13.2 \pm 5.98$ & $18.2 \pm 2.1$ & $15.56 \pm 3.25$ \\
\hline
\end{tabular}

